\title{
Questes
}

\section{La règle du jeu au Moyen Âge : «On ne peut bien sans regle ouvrer »}

Amandine Mussou et Laëtitia Tabard

\section{(2) OpenEdition}

1 Journals

\section{Édition électronique}

URL : http://journals.openedition.org/questes/662

DOI : 10.4000 /questes.662

ISSN : 2109-9472

Éditeur

Les Amis de Questes

\section{Édition imprimée}

Date de publication : 15 février 2010

Pagination : 4-29

ISSN : 2102-7188

\section{Référence électronique}

Amandine Mussou et Laëtitia Tabard, « La règle du jeu au Moyen Âge : « On ne peut bien sans regle ouvrer » », Questes [En ligne], 18 | 2010, mis en ligne le 04 septembre 2015, consulté le 23 septembre 2020. URL : http://journals.openedition.org/questes/662 ; DOI : https://doi.org/10.4000/questes.662 


\title{
Introduction : La règle du jeu au Moyen Âge : «On ne peut bien sans regle ouvrer»
}

\author{
Amandine MUSSOU et Laëtitia TABARD
}

Parce qu'il détend, divertit et distrait, le jeu appartient au domaine du loisir et constitue l'envers du travail productif. Jouer, ce pourrait être revenir à l'enfance, retrouver des gestes immémoriaux, se passionner de manière incompréhensible pour des enjeux dérisoires et oublier un instant le monde des activités sérieuses. Ainsi l'homme qui joue régresserait-il, dépenserait-il son énergie en pure perte. Aborder la question de la règle du jeu au Moyen Âge nous invite toutefois à privilégier ce qui, dans le jeu, semble au premier abord clairement déterminé et structurant, ce par quoi il échappe au domaine du temps perdu et de la gratuité : par la règle, le jeu devient d'une certaine manière un objet d'étude sérieux.

L'expression «règle du jeu» n'apparaît cependant, selon les lexicographes, qu'en $1538^{1}$. Est-il dès lors anachronique d'étudier les règles du jeu médiévales ? Le refrain de la ballade d'Eustache Deschamps l'affirme : «on ne peut bien sans regle ouvrer $»^{2}$; mais cette règle toute matérielle, «esquierre » ou « ligne », doit guider le trait du maçon, du charpentier et de «l'escripvain », et ne vaut pas comme métaphore pour le principe du jeu, seulement pour l'affaire autrement plus sérieuse qu'est le bon gouvernement. Que l'expression ne soit pas recensée dans les dictionnaires ne signifie pas que le jeu médiéval ne consiste qu'en un

\footnotetext{
${ }^{1}$ C'est ce qu'affirme le Dictionnaire historique de la langue française, sous la direction d'Alain REY, et les dictionnaires de l'ancien et du moyen français ne recensent pas cette expression.

2 Ballade MCCCLXIV, Euvres complètes de Eustache Deschamps, publ. d'après le ms de la Bibliothèque nationale par le marquis de Queux de Saint-Hilaire, Paris, FirminDidot, 1878-1903, tome VII, p. 203-204.
} 
divertissement totalement libre que n'encadrerait aucun principe préalable, aucun ensemble plus ou moins mouvant de lignes de conduite à respecter. Interroger les pratiques ludiques médiévales à partir de leurs règles, c'est poser la question du rôle définitoire ou non de la règle et donc envisager le fonctionnement interne du jeu, mais c'est également appréhender le rapport du jeu à toute forme de régulation externe.

\section{Ce que jouer veut dire ${ }^{3}$}

Définir le jeu est une entreprise difficile. Où commence le jeu et où s'arrête-t-il ? Quel est le point commun entre le jeu de dames et le jeu de l'acteur? Entre la marelle et le jeu du roi qui ne ment? La polysémie est telle que le terme est qualifié par certains de «poubelle linguistique ${ }^{4}$. Ainsi le français place-t-il sous le terme de jeu ce pour quoi l'anglais dispose de deux mots, play et game. Dans le jeu, on se soumet à un ensemble de règles, qui définissent un temps, un espace, et codifient des gestes, pour aboutir à la désignation d'un gagnant et d'un perdant : le jeu correspond alors à ce que l'on nomme game en anglais. Mais le mot peut également désigner l'action de jouer et se réfère alors à une pratique moins définie, une activité ludique libre et sans règle: c'est ce que l'anglais désigne par le terme play. On adopte un comportement dénué de sérieux, décalé, qui instaure un espace de fiction et se démarque ainsi de la vie courante. On peut d'ailleurs jouer à un jeu précis comme à autre chose (à la guerre, à faire les fous...). Le mot jeu réunit en français moderne ces deux acceptions, d'où son ambiguïté. D'après Jean-Michel Mehl, le point commun peut être trouvé dans l'idée de mouvement: le «jeu de l'acteur»

\footnotetext{
3 Nous nous inspirons ici largement des analyses de Jean-Michel MEHL dans l'introduction de son ouvrage Les Jeux au royaume de France du XIII au début du XVI siècle, Paris, Fayard, 1990, «Le jeu : un objet d'histoire », p. 14 sqq.

${ }^{4}$ Susanna Millar, La Psychologie du jeu chez les enfants et les animaux, Paris, Payot, 1971 ( $1^{\text {ère }}$ édition en anglais en 1968), p. 7.
} 
désigne sa manière de se mouvoir et de se déplacer, le joueur d'échecs déplace des pièces sur l'échiquier ${ }^{5}$. Le jeu est un ensemble de gestes, de mouvements, qui s'enchaînent à la fois librement - « il y a du jeu »-et en suivant des règles - l'image du « jeu des forces sociales » se fonde ainsi sur le sens de «mouvement contraint». Le mot jeu conjoint alors deux sens apparemment contradictoires.

Il y a donc lieu de se demander si l'on peut limiter le sens de jeu à une activité plaisante qui suit des règles. Mieux vaut peut-être distinguer seulement trois acceptions principales qui correspondent à une continuité de pratiques, de la plus contrainte à la plus libre : le jeu désigne le matériel ludique concret, le système de règles que s'impose le joueur, et enfin la pratique ludique elle-même - l'action de jouer, où l'on passe du game au play. Mais ces différents sens dérivent en fait du dernier : tout matériel peut devenir ludique dans la pratique, la règle ne crée le jeu que si le joueur y adhère, sans quoi elle n'est que pesante contrainte, et l'action de jouer se définit moins par le geste que par l'intention, caractère spécifique de l'acte ludique mais qui demeure par nature insaisissable ${ }^{6}$. Tout semble alors pouvoir devenir jeu, et il faut prendre en compte l'ensemble des manifestations ludiques pour cerner précisément le sens de ce terme.

Pour Johan Huizinga, le jeu est la forme première de la culture, et c'est dans l'acte ludique lui-même que s'élaborent à l'origine la poésie, la philosophie, les structures judiciaires, par exemple. Comme « action libre, sentie comme "fictive" et située en dehors de la vie courante », le jeu est « capable néanmoins d'absorber totalement le joueur », et « se déroule avec

\footnotetext{
${ }^{5}$ Jean-Michel MeHL, Les Jeux au royaume de France, op. cit., p. 15.

${ }^{6}$ Jean-Michel MeHL (dir.), Jeux, sports et divertissements au Moyen Âge et à l'Âge classique, Paris, éditions du Comité des Travaux Historiques et Scientifiques (CTHS), 1993, introduction, p. 7-8 notamment.
} 
ordre selon des règles données $»^{7}$, par quoi il conjoint sentiment d'irréalité et sentiment du sérieux. Reprenant et corrigeant son prédécesseur, Roger Caillois parvient à la définition analytique suivante. Le jeu est une activité libre (à laquelle le joueur ne saurait être obligé sans que le jeu perde aussitôt sa nature de divertissement attirant et joyeux), séparée (circonscrite dans des limites d'espace et de temps précises et fixées à l'avance), incertaine (dont le déroulement ne saurait être déterminé ni le résultat acquis préalablement, une certaine latitude dans la nécessité d'inventer étant obligatoirement laissée à l'initiative du joueur). Le jeu est par ailleurs improductif (il ne crée ni bien, ni richesse, ni élément nouveau d'aucune sorte), réglé (c'est une activité soumise à des conventions qui suspendent les lois ordinaires et qui instaurent momentanément une législation nouvelle, qui seule compte), fictif (il est accompagné d'une conscience spécifique de réalité seconde ou de franche irréalité par rapport à la vie courante $)^{8}$.

L'un des critères de définition du jeu réside donc dans les règles qui l'instituent comme tel. Selon Roger Caillois, ce sont des règles «précises, arbitraires, irrécusables, qu'il faut accepter comme telles et qui président au déroulement correct de la partie $»^{9}$. Loin de n'être qu'un élément parmi d'autres, la règle constitue donc le jeu: conventionnelle, elle crée un espace de fiction, séparé de la vie courante. Roger Caillois reconnaît cependant que beaucoup de jeux ne comportent pas de règles : il n'existe pas de règles pour jouer à la poupée ou au soldat, autrement dit pour les jeux qui supposent une libre improvisation. Néanmoins, la fiction du comme si remplace la règle et remplit la même fonction. Le théoricien

\footnotetext{
7 Johan Huizinga, Homo ludens, Essai sur la fonction sociale du jeu, Paris, Gallimard, 1972 ( $1^{\text {ère }}$ édition en 1938), p. 35.

${ }^{8}$ Roger CAILlois, Les Jeux et les hommes. Le masque et le vertige, Paris, Gallimard, 1967 (édition revue et commentée, $1^{\text {ère }}$ édition en 1958), p. 42-43.

${ }^{9}$ Pour tout ce passage, ibid., p. 38 sqq.
} 
distingue alors deux catégories : les jeux pour lesquels on joue pour de bon, dont le respect des règles nous sépare de la vie courante (les échecs ou le polo par exemple); ceux qui consistent à imiter la vie tout en ayant conscience de l'irréalité foncière du comportement alors adopté (jouer à la dînette, jouer aux gendarmes...). «Ainsi, les jeux ne sont pas réglés et fictifs. Ils sont plutôt ou réglés ou fictifs $»^{10}$. Cette première distinction permet à Roger Caillois de mettre en place sa classification des jeux en quatre catégories: agôn, renvoyant aux jeux de compétition, alea, désignant les jeux fondés sur une décision qui ne dépend pas du joueur, mimicry, pour définir les jeux consistant à devenir soi-même un personnage illusoire et à se conduire en conséquence, et ilinx, rassemblant les jeux reposant sur « la poursuite du vertige $»^{11}$.

On a reproché à Johan Huizinga de soutenir que tout découlait du jeu et $d$ 'adopter une définition beaucoup trop large ${ }^{12}$. Plus généralement, selon le sémiologue Louis-Jean Calvet, c'est le type d'approche employé par Johan Huizinga et Roger Caillois qui pose problème : il se fonde en effet sur un système linguistique précis non nécessairement transposable dans une autre langue. Ainsi, l'italien et l'anglais n'emploient pas le terme de jeu pour parler du « jeu de l'acteur ». Dans sa classification des jeux, Roger Caillois a donc fait «œuvre de francophone»: est pour lui jeu ce que le français classe comme tel ${ }^{13}$. La polysémie du verbe français jouer n'est pas universelle et l'auteur reproche aux deux théoriciens du jeu d'avoir été victimes d'une «illusion sémantique » imputable aux différents sens du verbe jouer en français. Louis-Jean Calvet conclut donc : «Dans tous les

\footnotetext{
${ }^{10}$ Ibid., p. 41.

${ }^{11}$ Ibid., p. 67. Pour cette classification, voir l'ensemble du chapitre II de la première partie.

${ }^{12}$ Voir par exemple Jacques HenRIOT, Le Jeu, Paris, PUF, 1969, p. 6 notamment.

${ }^{13}$ Louis-Jean CALVET, Les Jeux de la société, Paris, Payot, 1978, p. 12.
} 
cas, donc, le jeu n'existe pas, il n'y a que les jeux, et les diverses façons de les pratiquer $»^{14}$.

Ce reproche n'enlève rien à la validité de la définition de Roger Caillois en ce qui concerne les jeux en France. Pour la période médiévale, il est même nécessaire de donner à la notion une vaste extension, dans la mesure où l'ancien français regroupe sous le terme de jeu encore bien plus de pratiques que le français moderne. Le terme de jeu vient de jocus, et a un sens extrêmement vaste : employé tout d'abord en latin classique avec le sens spécifique de «plaisanterie», «badinage» (sens conservé par l'anglais joke), le terme au pluriel, joci, s'étend à toutes les distractions : chants, danses, concours, ébats, amusements... ${ }^{15}$ Au départ lié à la parole, le jocus étend finalement son domaine de signification et supplante l'autre terme latin, ludus, lui-même très polysémique, dans la plupart des langues romanes. Jocus se réfère à un comportement moins défini que ludus, mais guidé par l'intention de se divertir. Le jongleur, joculator, est celui qui divertit; il joue de son corps, par les gestes et les postures, et de la parole, dans la diction et le chant.

Ce sens très large se retrouve en ancien français dans l'emploi absolu du verbe jouer. Dans le Dit de Poissy de Christine de Pizan, la narratrice, qui veut $«$ jouer ${ }^{16}$, choisit de rendre visite à sa fille en compagnie de ses amis. Le jeu est ici un moment de joie partagée, qui s'oppose au sérieux, au respect des règles de la vie courante. Il appartient au domaine du divertissement, au sens large. En ce sens, le jeu s'oppose à la règle. Lorsque Christine de Pizan définit «l'ordre de vivre en quoy [la noble ${ }^{14}$ Ibid., p. 16.

${ }^{15}$ Jean-Michel Mehl cite par exemple Horace, Odes, III, 21 : «O nata mecum consule Manlio,/ seu tu querellas sive geris jocos/ seu rixam et insanos amores/ seu facilem, pia testa, somnum [...] » (Amphore qui naquis en même temps que moi/ Que tu portes en toi les plaintes, les loisirs,/ Les rixes, les amours insensés ou encore/ Un facile sommeil, ô toi ma bienfaisante $[\ldots])$.

${ }^{16}$ Christine De Pizan, Le Dit de Poissy, Barbara Altmann (éd.), The Love Debate poems of Christine de Pizan, Gainesville, University Press of Florida, 1998, v. 41. 
dame] vouldra estre riglee $»^{17}$, elle exclut précisément les jeux de ce qui doit être codifié :

«Quant est des autres esbatemens a quoy dames seulent prendre plaisir, si comme d'aller a la chace aucunes fois, voler en riviere, ou danser aux joennes, ou autres jeux, ces choses nous ne mettons point en l'ordre de notre discipline ou enseignemens, car nous les laissons en la distribucion et vouloir de leurs mariz, et du leur aussi, desquelles choses aucune licence puet bien estre donnee en temps et en lieu, meismes aux dames tres vertueuses, sans mesprendre, mais que ce soit sans trop et que mesure y soit gardee. $»^{18}$

C'est dire que les jeux se situent en dehors du champ des obligations et des règles de conduite habituelles, ce qui n'exclut pas, bien sûr, que d'autres règles s'appliquent, lorsqu'on chasse ou on danse. Mais force est de constater que la notion de "règle » est du côté de la discipline et doit guider le comportement et les pensées de la femme de bien.

On a le sentiment que l'essentiel du jeu tient non pas à la règle mais à la séparation d'avec la vie courante ${ }^{19}$. Le jeu, compris comme activité se déployant dans un espace fictif, séparé, donne lieu à de très nombreuses expressions : on rappelle à l'ordre en disant ce n'est pas geu, c'est-à-dire «ce n'est pas pour rire », ou bien à l'indulgence en demandant de prendre à jeu, c'est-à-dire de considérer comme une plaisanterie. L'art des jongleurs est celui du jeu et la représentation dramatique se nomme jeu. Comme les autres termes exprimant le divertissement, deporter, deduire, esbatre, esbanoier, avec lesquels il commute aisément même si on l'emploie plus volontiers seul, le verbe jouer s'emploie pour une activité

${ }^{17}$ Christine De PIZAN, Le Livre des trois vertus, Charity Cannon WILlard et Eric Hicks (éd.), Paris, Champion, 1989, p. 43.

${ }^{18}$ Ibid, p. 51.

${ }^{19}$ Cette séparation ne saurait être une frontière étanche, ainsi que le rappelle Daniel Poirion : les analyses de Johan Huizinga sur la pénétration du jeu et du spectacle dans toutes les sphères de la vie sociale montrent qu'une conception ouverte du jeu est nécessaire pour aborder les pratiques médiévales, voir Daniel POIRION, Le Poète et le prince. L'évolution du lyrisme courtois de Guillaume de Machaut à Charles d'Orléans, Paris, PUF, 1965/ Genève, Slatkine Reprints, 1978, p. 73-74. 
qui procure de la joie ${ }^{20}$ - d'où peut-être son sens érotique - et qui rompt avec la vie courante, comme l'indique dans les autres verbes l'emploi des préfixes de- ou ex-. Le jeu médiéval, dont la définition semble encore plus souple et plus fuyante que celle du jeu au sens moderne, ne se plie pas facilement à nos règles.

\section{Regards modernes sur le jeu au Moyen Âge}

L'écart entre les catégories modernes et les pratiques médiévales si diverses regroupées sous le terme de jeu constitue l'un des obstacles à l'étude du domaine ludique, alors que la société médiévale est très joueuse. Jean-Michel Mehl parle, pour les derniers siècles du Moyen Âge, de véritable « croissance ludique ${ }^{21}$.

L'ouvrage de cet historien marque un tournant, en proposant une synthèse sur la période : excluant de son étude les catégories de l'ilinx et de la mimicry, soit tout ce qui concerne le jeu théâtral, le jeu carnavalesque ou le jeu musical, il dresse un panorama instructif de l'historiographie des jeux de société et des sports. S’il décrit les jeux, il prend également en compte la manière dont la société de la fin du Moyen Âge les considère et écarte les préjugés modernes sur la question. Jean-Michel Mehl relève d'emblée la place restreinte qu'occupent les jeux dans l'historiographie des $\mathrm{XIX}^{\mathrm{e}}$ et $\mathrm{XX}^{\mathrm{e}}$ siècles et montre que la part ludique de la vie des hommes échappait jusqu'à peu à l'analyse historique. Le peu de mémoire que les sociétés ont de leurs jeux et le peu de valeur qu'elles leur accordent expliquent en partie

\footnotetext{
${ }^{20}$ Globalement, comme le remarque Philippe Ménard, l'idée de plaisir prédomine dans le lexique du divertissement, et non l'idée d'un loisir s'opposant aux activités. Jeu veut aussi dire « plaisir », « joie », et désigne le contraire de la détresse dans l'expression $n{ }^{\prime} i$ a ne gieu ne ris, que l'on peut traduire à peu près par «ce n'est pas la joie ", voir Philippe Menard, "Le concept de loisir au Moyen Âge», in Jean-Marie ANDRE, Jacqueline DANGel, Paul DemOnt (dir.), Les Loisirs et l'héritage de la culture classique. Actes du XIII Congrès de l'Association Guillaume Budé (Dijon, 27-31 août 1993), Bruxelles, Latomus, Revue d'études latines, 1996, p. 455-469.

${ }^{21}$ Jean-Michel MeHL, Les Jeux au royaume de France, op. cit., p. 458.
} 
que ce domaine ait été délaissé longtemps par les médiévistes : on dispose ainsi de sources très lacunaires pour étudier les jeux médiévaux. Hormis quelques rares ouvrages 22 , les véritables travaux sur l'histoire des jeux étaient des monographies portant sur tel ou tel dispositif ludique ${ }^{23}$.

Jean-Michel Mehl commence son étude au XIII ${ }^{\mathrm{e}}$ siècle, la faiblesse de la documentation rendant impossible toute étude sur la période antérieure à 1200. Il se fonde, pour mener à bien son entreprise, sur différents types de sources, dont les lettres de rémission, actes par lesquels le roi de France ou un grand seigneur, par arrêté de justice, fait remise entièrement ou partiellement de sa peine à un condamné. Beaucoup de ces lettres font état de jeux comme incidents à l'origine de la condamnation. Il faut cependant se méfier de ces sources, souvent simplificatrices. Par ailleurs, l'historien souligne la difficulté de dresser un tableau socioprofessionnel des joueurs à partir de ces lettres : par exemple, on risque de considérer que les étudiants subissaient une plus grande attirance pour les jeux car beaucoup de délinquants tentaient de se faire passer pour des clercs afin de bénéficier de leurs privilèges. Les comptabilités domestiques et les inventaires des biens mobiliers fournissent d'autres sources, à manier cependant avec précaution puisqu'on connaît mieux les gestes quotidiens des rois et des reines, et donc leurs jeux, que ceux du peuple et de la bourgeoisie. Enfin, toute une documentation législative, réglementaire ou répressive, comme une législation ecclésiastique formelle interdisant aux

\footnotetext{
${ }^{22}$ Voir Jean-Jules JusSERAND, Les Sports et les jeux d'exercice dans l'ancienne France, Paris, Champion, 1901/ Genèvre, Slatkine Reprints, 1986.

${ }^{23}$ Dès 1895, un article de Fritz StrohmeYER est consacré au jeu d'échecs («Das Schachspiel im Altfranzosischen », Abhandlungen Herrn Prof. Dr. Adolf Tobler, Halle, Niemeyer, 1895, p. 381-403) et en 1913, Franz SEMRAU s'intéresse au jeu de dés (Würfel und Würfelspiel im alten Frankreich, Halle, Druck von E. Karras, 1909). Ces études reposent uniquement sur des sources littéraires. En 1913 paraît A History of chess, de Harold James Ruthven MurRaY (Oxford, Clarendon Press), véritable somme qui demeure inégalée pour les autres jeux. Même plus tard, hormis Jean VERDON, Les Loisirs en France au Moyen Âge (Paris, Jules Tallandier, 1980), peu de travaux sont consacrés au jeu médiéval.
} 
prêtres, moines et évêques de jouer, permet également de faire du jeu un objet d'histoire. Le caractère répétitif de cette législation montre que les ecclésiastiques respectaient peu cette interdiction et cette étude informe donc sur les pratiques ludiques ${ }^{24}$.

L'historien se fonde également sur les fictions littéraires, tout en relevant certains écueils auxquels il se trouve confronté. Dans les récits médiévaux, l'aristocrate semble être celui qui joue. Dans quelle mesure l'état de la noblesse était-il réellement perçu comme lié au jeu, en dehors de ce qui peut n'être qu'un cliché littéraire ? Cette passion des nobles pour les jeux qui transparaît dans les chansons de geste, la lyrique et les romans est toutefois confirmée par les comptabilités domestiques des grands personnages. La concordance des sources souligne bien les liens privilégiés qui existaient entre l'aristocratie et le jeu. Comble de cette passion, certains adoptaient un instrument de jeu en guise d'insigne ou d'armoiries: les hommes du bailli Pierre de Hangenbach en 1475 portaient sur leur livrée un dé brodé accompagné des mots « Je passe ${ }^{25}$. Toutefois, d'autres types de problèmes sont liés à ces sources : à en croire les témoignages littéraires, les échecs étaient le jeu le plus pratiqué par l'ensemble de la noblesse. Néanmoins, en raison du coût que pouvait représenter l'acquisition d'un bel échiquier ainsi que du rôle littéraire du jeu d'échecs et des symboles dont il était porteur, cette omniprésence des soixante-quatre cases n'était certainement pas aussi marquée. La prégnance de l'échiquier dans la vie des aristocrates ne serait donc parfois qu'un stéréotype littéraire sans rapport avec la réalité - les formules récurrentes dans les textes décrivant un noble jouant «a dés, as tables, as eschés » seraient la marque de ce cliché $^{26}$. Cependant, le public à qui s'adressent ces textes devait quand

\footnotetext{
${ }^{24}$ Jean-Michel MeHL, Les Jeux au royaume de France, op. cit., p. 214.

${ }^{25}$ Ibid., p. 197.

${ }^{26}$ Ibid., p. 198.
} 
même connaître les échecs, sans quoi on comprendrait mal la permanence de ce topos.

Dans le domaine des études littéraires, on peut déceler un retard dans la reconnaissance de la valeur des activités ludiques, sans doute parce que la littérature ludique et récréative est soupçonnée de n'être qu'un divertissement, et que cette conception qui fait de l'œuvre littéraire un instrument dont use une société choque notre conception de l'autonomie de l'art et de la dignité des belles-lettres. Soupçonné de gratuité, de frivolité, le jeu semble réduire l'œuvre à sa fonction sociale. Les travaux de Mikhaïl Bakhtine, pour controversés qu'ils soient, ont ouvert un champ de recherche dans cette partie de la littérature dite «récréative », considérée comme une émanation de la culture populaire du Moyen Âge. À ce titre, elle est révélatrice d'une vision du monde et non d'une mentalité archaïque ou grossière. Il n'existe néanmoins pas d'équivalent de la synthèse de JeanMichel Mehl en littérature et les ouvrages concernant les jeux de société ou les sports sont bien plus ponctuels et concernent en général un jeu en particulier $^{27}$, comme par exemple, un ouvrage récent sur l'utilisation des échecs dans la littérature médiévale ${ }^{28}$.

Contrairement aux recherches menées par les historiens, les travaux littéraires s'intéressent davantage au jeu dans la dimension très large que lui accorde Huizinga, comme une forme de culture. Le jeu de société, en tant que dispositif matériel visant à divertir, est moins analysé que le jeu au sens de série de codes sociaux dont on souligne l'importance civilisatrice. Daniel Poirion, dans Le Poète et le prince, insiste sur cette notion de jeu « qui apparaît inévitablement quand on veut définir l'attitude aristocratique

\footnotetext{
${ }^{27}$ Hormis Jean-Marie ANDRE, Jacqueline DANgel, Paul Demont (dir.), Les Loisirs et l'héritage de la culture classique, op. cit.

${ }^{28}$ Jenny ADAms, Power Play. The Literature and Politics of Chess in the Late Middle Ages, Philadelphia, University of Pennsylvania Press, "The Middle Ages Series", 2006.
} 
devant le monde $»^{29}$. Le jeu organise la vie courtoise et c'est surtout à ce titre qu'il est étudié chez les spécialistes de la littérature.

Si Daniel Poirion montre que le développement de la vie de cour a favorisé depuis longtemps les jeux de société, il insiste beaucoup sur les modalités de cette vie, menée par une aristocratie qui se détourne du travail pratique, dans laquelle le poète puise son élan créateur. Il évoque ainsi les Jeux à vendre de Christine de Pizan $^{30}$, les concours poétiques à la cour de Charles d'Orléans... Chez Daniel Poirion, l'intérêt pour ces formes poétiques permet de repenser la valeur du jeu : la dimension ludique ne réduit pas ces textes, elle permet au contraire d'envisager cette production comme véritablement littéraire. Les travaux de Michèle Gally sur les jeuxpartis vont dans ce sens et insistent sur la présence d'échanges ludiques très formalisés en domaine courtois ${ }^{31}$.

C'est le jeu au sens de divertissement réglé, incarné dans des corps, qui intéresse les spécialistes de littérature médiévale davantage que les jeux de société et les sports. En d'autres termes, les études littéraires portent davantage sur la mimicry que sur l'agôn ou l'alea, ce qui peut être expliqué par la fertilité littéraire de tels jeux, qui produisent des œuvres, à la marge du théâtre et de la poésie : ces approches du jeu conduisent à un nouveau regard sur la règle, qui a en ce domaine valeur de support et non de carcan.

\section{Règles et réglementations}

Aussi importe-t-il de distinguer nettement la règle de la réglementation qui formule les interdits, alors même que l'on connaît bien mieux la législation que les principes mouvants et implicites qui organisent

\footnotetext{
${ }^{29}$ Daniel PoIRIOn, Le Poète et le prince, op. cit., p. 73.

${ }^{30} \mathrm{Au}$ cours de ces jeux, une dame lançait à un gentilhomme, ou inversement, le nom d'une fleur, ou d'un objet, et la personne interpellée devait répondre immédiatement par un compliment ou une épigramme rimée.

${ }^{31}$ Michèle Gally, Parler d'amour au puy d'Arras. Lyrique en jeu, Orléans, Paradigme, 2004.
} 
les jeux médiévaux. Aucun document conservé ne consigne expressément les règles suivies par les joueurs médiévaux, qui ne sont donc reconstituées que partiellement, à partir de traités tardifs ou de bribes d'informations tirées de textes littéraires. Jusqu'en 1650, elles ne sont pas encore écrites et se constituent au travers d'une multitude de coutumes qui vont peu à peu s'harmoniser. Mais, au cours du Moyen Âge, il n'est pas de jeu qui échappe à une définition plus précise ou qu'un ensemble de dispositions réglementaires ne vienne corseter ${ }^{32}$. On assiste à une domestication des jeux par une forme d'encadrement, par la moralisation ainsi que par l'élaboration de mesures visant à les restreindre et à les contrôler.

Le jeu est considéré comme une invention diabolique par les moralistes depuis le haut Moyen Âge, comme peuvent l'illustrer les joueurs de dés qui jouent les vêtements du Christ ${ }^{33}$. Au XIII ${ }^{\mathrm{e}}$ siècle, le Speculum morale de Vincent de Beauvais recommandait de fuir les jeux et la compagnie des joueurs ${ }^{34}$. Le Livres dou tresor de Brunetto Latini et Le Mesnagier de Paris insistent sur les liens entre jeu et colère, luxure et avarice $^{35}$. À ces préjugés religieux et moraux s'ajoutent des préjugés politiques : le joueur de dés ne se muscle pas et donc ne se prépare pas à la guerre ! Mais le discours des théologiens, des canonistes et des prédicateurs ne permet pas de rendre compte à lui seul de la perception du jeu dans la

\footnotetext{
32 Jean-Michel MeHL, Les Jeux au royaume de France, op. cit., p. 464.

${ }^{33}$ Voir illustration 1, p. 28.

34 VINCENT DE BEAUVAIS, Speculum morale, Speculum quadruplex sive Speculum majus, Graz, Akademische Druck- u. Verlagsanstalt, 1964, t. III, p. 1361 sqq : «De ludis inhonestis ».

${ }^{35}$ Références citées par Jean-Michel MeHL, Les Jeux au royaume de France, op. cit., p. 323. Voir Brunet Latin, Li livres dou tresor, Polycarpe Chabaille (éd.), Paris, Imprimerie impériale, 1863, p. 292 : «porce que jeus decline maintes foiz en courrouz ou en deshonnor et est commencemens de luxure ». Voir également Le Mesnagier de Paris, texte édité par Georgina E. BRERETON et Janet M. FERRIER, traduction et notes par Karin UeltschI, Paris, Le Livre de Poche, "Lettres Gothiques », 1994, I, III, 83, p. 102 : « La .VI. ${ }^{\text {e }}$ branche d'avarice si est le hazart. Si est quant on joue aux dez pour gaignier l'argent d'autruy: et y a moult de barat, de couvoitise, et d'avarice et de decepcion ».
} 
société médiévale : tous, clercs ou laïcs, ne partageaient pas une attitude totalement négative. On peut ainsi parler d'un jeu toléré ${ }^{36}$, même si une ferme distinction demeure entre les jeux de hasard, condamnés, et les autres.

Peu à peu, l'utilité de certains jeux finit même par être reconnue. Cette évolution a sans doute à voir avec la réhabilitation de la notion de loisir assumée en grande part par la courtoisie. Guillaume de Lorris renverse par exemple la valeur du terme Oiseuse pour en faire une qualité courtoise ${ }^{37}$. Puis, aux $\mathrm{XIV}^{\mathrm{e}}$ et $\mathrm{XV}^{\mathrm{e}}$ siècles, ce sont les notions de recreacion et de passe-temps qui deviennent importantes. Le jeu est alors même recommandé dans certains ouvrages de bon gouvernement, comme dans Le Livre du corps de policie, où Christine de Pizan explique « comment il est loisible que le bon prince aprés grant labour prengne recreacion d'aucun esbatement $»^{38}$.

Le jeu est non seulement reconnu comme une nécessité, notamment pour les princes, mais il est également entendu comme un véritable support d'apprentissage. On connaît la fortune des échecs, considérés comme facilitant l'acquisition d'un savoir, de capacités intellectuelles et donc faisant partie de l'éducation de tout jeune aristocrate. Les échecs sont reconnus à la fin du Moyen Âge à condition d'avoir rompu toute attache avec l'argent et le hasard. C'est ainsi que sur l'échiquier du traité de Jacques de Cessoles, le De ludo scacchorum, premier ouvrage de moralisation et d'allégorisation systématique des échecs, chaque pièce est

\footnotetext{
36 Jean-Michel Mehl intitule l'une de ses sections «Le jeu toléré », Les Jeux au royaume de France, op. cit., p. 325 sqq.

37 Voir sur ce point l'article de Philippe MENARD, «Le concept de loisir au Moyen Âge ", art. cit.

38 Christine DE PIZAN, Le Livre du corps de policie, édition critique par Angus J. KenNEDY, Paris, Honoré Champion, 1998, p. 53-54. Le terme de jeu apparaît à la fin de ce passage faisant l'éloge du temps libre : «Car en temps et en lieu se jouaient ensemble en jeux honnestes et beaux ».
} 
associée à une fonction sociale ${ }^{39}$ : 1'un des pions figure un joueur ayant pour attributs de l'argent et des dés, rappelant ainsi en creux l'une des règles originelles des échecs qui préconisait l'usage de dés pour faire avancer les pièces - règle que l'Église avait toujours fermement condamnée et qui disparaît peu à peu.

C'est donc la notion de mesure qui paraît ici importante : l'attitude ludique est condamnée si elle donne lieu à des excès. La violence liée au jeu est attestée par de nombreux témoignages : en 1530, dans une taverne flamande, Jean Amoen utilise une partie de dés pour chercher querelle à un joueur qui avait blessé son cousin et le tuer ${ }^{40}$. C'est ainsi que 1'on peut analyser, avec Daniel Poirion, le passage au XIV ${ }^{\mathrm{e}}$ siècle, des tournois, véritables batailles où il en va de la vie même des participants, aux joutes où le théâtre 1 'emporte sur le sport $^{41}$. On se sert alors de la joute pour paraître plutôt que pour affirmer sa prouesse : c'est la métamorphose de l'agôn en mimesis, de la compétition au simulacre, de la chevalerie de fait à la chevalerie factice. Cette spectacularisation peut également être entendue comme une canalisation de la violence par le jeu, ce dont témoigne une volontaire mainmise des autorités sur les pratiques ludiques.

\footnotetext{
${ }^{39}$ Pour le texte latin, voir l'édition d'Ernst KÖPKE in Jahresbericht der Mittheilungen aus den Handschriften der Ritter-Akademie zu Brandenburg, II, Brandenburg, 1879. Pour une traduction médiévale, voir JACQUES DE CESSOLES, Le Jeu des eschaz moralisé, traduction de JEAN FERRON, édition publiée par Alain COLLET, Paris, Honoré Champion, "Classiques français du Moyen Âge», 1999. Pour une traduction en français moderne, voir JACQUES DE CESSOLES, Le Livre du jeu d'échecs ou la société idéale au Moyen Âge, XIII siècle, traduit et présenté par Jean-Michel MEHL, Paris, Stock, "Moyen Âge », 1995. Voir également la thèse de doctorat de Jean-Michel MeHL, Jeu d'échecs et éducation au XIII siècle. Recherches sur le "Liber de moribus " de Jacques de Cessoles, Université des sciences humaines de Strasbourg, 1975.

${ }^{40}$ Voir à ce sujet Jean-Michel MeHL, Les Jeux au royaume de France, op. cit., p. 305.

${ }^{41}$ Daniel PoIrIOn, Le Poète et le prince, op. cit., p. 78.
} 
La réglementation des jeux par les autorités touche tout d'abord leur fabrication $^{42}$. Ainsi était interdite la confection de dés longnés, c'est-à-dire « frottés à la pierre d'aimant », mepoints, c'est-à-dire « pipés », et plombés, reconnaissance implicite de larges pratiques frauduleuses. Avec ce type de réglementation, s'affirme une tentative d'encadrement du jeu, d'autant plus diabolique qu'il s'en remet au hasard et qu'il suscite la tromperie. Les témoignages de fraude sont nombreux, comme dans le Dit du gieu de dez, où Eustache Deschamps évoque un autre type d'escroquerie, qui consiste à faire bouger la table :

«L'un des joueurs gette ambesas*, *double as, coup du jeu de dé

Et vit que la table trembla;

Le coup pert, puis regardé l'a,

Et regniant Dieu et sa mere

Disant : « de male mort amere

Puist mourir cilz qui est au bout !

Sire, vous m'avez fait du tout

Perdre le mien a ceeste fois :

Vous n'estes mie bien courtois. ${ }^{43}$

Les relations entre les autorités et le jeu sont d'une manière générale tumultueuses $^{44}$. Souvent, l'acte répressif constitue la première trace tangible de l'existence du jeu. Le pouvoir royal peut intervenir, mais l'action n'est pas très fréquente à ce niveau: on recense une douzaine d'ordonnances royales sur le jeu entre 1254 et 1537. Des condamnations au niveau municipal sont plus nombreuses; un arsenal de mesures émane également de l'autorité spirituelle et est destiné aux clercs, tout en ne

\footnotetext{
${ }^{42}$ Le Livre des métiers du prévôt de Paris Étienne Boileau, élaboré à partir de 1258 , présente notamment les statuts des tabletiers, qui fabriquaient les tabliers d'échecs, de dames ou de trictrac, et ceux des déciers, qui tenaient profession de «faiseurs de dés à tables et à eschiés, d'os et d'ivoire, de corne et toute autre matière », voir Le Livre des métiers d'Étienne Boileau, René DE LESPINASSE et François BONNARDOT (éd.), Paris, Imprimerie nationale, 1879.

43 Eustache Deschamps, Dit du gieu de dez, in CEuvres complètes de Eustache Deschamps, éd. cit., tome VII, p. 256.

${ }^{44}$ Voir à ce sujet Jean-Michel MeHL, Les Jeux au royaume de France, op. cit., p. 339 $s q q$.
} 
négligeant pas les laïcs. Les législateurs médiévaux ont largement puisé dans le droit romain qui s'est très tôt attaqué aux jeux de hasard, le complétant par les règles de l'Église. En dépit de ces contraintes judiciaires, les jeux de hasard ont toujours été pratiqués : si l'on ne dispose d'aucun moyen précis pour évaluer l'efficacité de ces lois, tout suggère qu'elles n'avaient qu'un impact limité. D'autant plus que l'attitude des pouvoirs face aux jeux n'est pas monolithique. Certes le jeu reste illicite, mais les autorités sont de plus en plus conscientes de leurs limites dans cette répression. On passe finalement de la répression $d u$ jeu à la répression des jeux. Les grands vainqueurs de cette évolution sont les jeux sportifs, comme «recreacion» physique. S'établit ainsi une hiérarchie: jeux sportifs et intellectuels sont non seulement tolérés et valorisés, mais encore replacés à l'intérieur d'un discours pédagogique. Ce sera surtout le cas à la Renaissance ; si Rabelais sourit de la liste des jeux du jeune Gargantua, il ne les élimine pas de son nouveau programme d'éducation.

L'insertion du jeu dans une pratique pédagogique pourrait cependant détruire ce qui le fonde. Dans quelle mesure la canalisation du jeu par les autorités morales n'en restreint-elle pas la dimension ludique ? Les Eschés amoureux, texte en vers de la fin du $\mathrm{XIV}^{\mathrm{e}}$ siècle, utilisent le support ludique dans un but clairement didactique ${ }^{45}$. Une partie d'échecs allégorique est décrite - du moins les neuf premiers échanges ainsi que les cinq derniers - et il est vraisemblable que la lecture d'un tel texte nécessitait la présence d'un précepteur capable de montrer sur un échiquier

\footnotetext{
45 Aucune édition intégrale des 30000 vers des Eschés amoureux n'est à ce jour disponible. Pour un accès au premier tiers du texte, voir Christine KRAFT, LiebesgartenAllegorie der "Echecs amoureux », Kritische Ausgabe und Kommentar, Frankfurt am Main, Peter Lang, 1977, et Gianmario RAIMONDI, "Les Eschés amoureux. Studio preparatorio ed edizione (I. vv. 1-3662)», Pluteus, 8-9 (1990-1998), Alessandria, Edizioni dell'Orso, p. 67-241 et «Les Eschés amoureux. Studio preparatorio ed edizione (II. vv. 3663-5538)», Pluteus, 10 (1999-2000), Alessandria, Edizioni dell'Orso, 2007, p. 39-158.
} 
réel les déplacements des pièces et leurs significations ${ }^{46}$. Le choix de l'échiquier d'amour ${ }^{47}$ sert ici d'appât pour livrer sous forme allégorique des règles de conduite courtoises, mais également pour délivrer des conseils de bon gouvernement, puisque la suite du texte repose notamment sur la traduction d'un miroir des princes ${ }^{48}$. Cet exemple rend compte de l'insertion du jeu dans un système didactique et permet de s'interroger sur la transition du jeu au sérieux, sur la manière dont un divertissement collectif se fige ou non, par la réglementation qui l'organise et qui l'encadre, en un rituel qui lui confère dignité et reconnaissance sociale.

\section{Du jeu au sérieux}

S'il est un domaine dans lequel une règle du jeu existe avant la réglementation, c'est bien celui du théâtre. La notion de jeu se révèle en effet la plus opératoire pour aborder le théâtre médiéval. Le terme de « jeu théâtral » est préféré à celui de «théâtre » par Jean-Pierre Bordier, parce que cette notion dépasse les distinctions modernes et permet d'aborder le phénomène théâtral au Moyen Âge en tenant compte de la spécificité de ses pratiques. Il faut « accepter au départ l'idée que des frontières indécises et des marges profondes sont précisément un caractère essentiel du jeu médiéval ${ }^{49}$. Il faut également rappeler que le théâtre médiéval reste un divertissement ouvert, même lorsqu'il se veut édifiant. La représentation théâtrale n'est alors pas totalement distincte du jeu de société : celui-ci

\footnotetext{
${ }^{46}$ C'est ce qu'affirment les éditeurs du commentaire en prose des Eschés amoureux, dans l'introduction de leur édition du Livre des eschez amoureux moralisés, ÉVRART DE CONTY, Le Livre des eschez amoureux moralisés, édition de Françoise GUICHARDTESSON et de Bruno Roy, Montréal, Ceres, « Bibliothèque du Moyen Français », 1993, introduction, p. LXII.

${ }^{47}$ Voir illustration 2, p. 29.

${ }^{48}$ Le poème adapte en effet ensuite le De regimine principum de Gilles de Rome.

49 Jean-Pierre Bordier, Stéphanie Le Briz-Orgeur, Gabriella PARussa (dir.), Le Jeu théâtral, ses marges, ses frontières, Actes de la deuxième rencontre sur l'ancien théâtre européen de 1997, Paris, Champion, «Le Savoir de Mantice », 1999, présentation de Jean-Pierre Bordier, p. 9.
} 
ouvre la possibilité d'un espace fictif de convention, où gestes et éventuellement paroles sont codifiés, et où chaque participant joue un rôle. Selon les catégories modernes, on se situe ici au seuil du théâtre, mais non au cœur de l'art dramatique, dans la mesure où le public n'est pas fondamentalement distinct des acteurs, ce qui ménage au sein de toute représentation une part d'improvisation ou de jeu avec le public. Cependant, force est de constater que là où les catégories modernes distinguent des champs culturels distincts, le Moyen Âge identifie une même pratique, et nomme jeu ce que nous appelons théâtre: pour reprendre les mots de Jean-Pierre Bordier, les spectateurs «n'étaient pas encore expulsés derrière le "quatrième mur" et l'impérialisme de l'illusion n'avait pas encore éliminé la recherche de la connivence $»^{50}$.

Comme c'est le cas pour les autres jeux, on réglemente le divertissement théâtral afin d'éviter les débordements; mais la réglementation confère à la scène le statut d'espace sacralisé, où se déploie une fiction que le public est invité à admirer de loin. La récurrence des interdictions en dit long sur les habitudes médiévales. L'ordonnance de police prise à Tournai en 1550 témoigne des habitudes d'un public prompt à participer :

«1) Qu'aucune personne quelle qu'elle soit, ne se permette de crier, huer, ni jeter de quelque manière que ce soit après les joueurs, de même quand ils feront les dits jeux, sous peine d'amende d'un carolus d'or.

2) Que nul ne se permette de vouloir entrer dans le local des jeux sans payer le droit d'entrée qui se percevra aux portes et qu'aucune personne ne se permette d'injurier les percepteurs du droit d'entrée sous peine de ladite amende.

3) Que personne ne se permette de lâcher ses eaux sur les estrades, ni sur les bonnes gens qui seront assis dessous, sous peine de deux carolus d'or.

${ }^{50}$ Ibid., p. 15. 
4) Que personne ne se permette de se battre ou de se quereller pendant les dits jeux, sous peine de ladite amende de deux carolus. $[\ldots] » 51$

Ce type de documents témoigne-t-il du passage à une autre conception de la représentation, moins ludique? L'intervention d'une autorité extérieure dans le «jeu par personnage » transforme de fait le jeu en spectacle, assure l'autonomie d'un espace de fiction, mais le dénature : il ne s'agit plus d'un jeu où chacun participe en se fondant sur des règles librement consenties. On est passé à une autre théâtralité, fondée sur l'illusion qu'il ne faut pas briser, et sur la mimesis. Ce changement est illustré par les dialogues étudiés par Jean-Claude Aubailly. Par rapport aux monologues dramatiques, qui privilégient l'interaction ludique entre un personnage parodique et le public, les dialogues déplacent l'intérêt de la salle à la scène où l'action se déroule : " cette "distanciation" nécessaire à tout acte qui de jeu veut devenir théâtre, est rendue possible parce que le heurt de deux psychologies véritables suffit à remplir la scène $»^{52}$.

A contrario, on pourrait dire que le jeu suppose une attitude particulière à l'égard des règles, considérées simplement comme des supports d'improvisation ou même d'imitation parodique. Ainsi, les « jeux à vendre », illustrés notamment par Christine de Pizan, unissent dans une forme les voix de deux participants, l'un donnant la première rime avant que le second ne complète le distique par un vers, en suivant simplement le principe poétique de la rime. Toute une partie de la littérature courtoise, qui tient à la sphère ludique, semble reposer plus précisément sur le détournement de règles, que ce soit les jeux-partis des trouvères, les tensons et partimens des troubadours, les débats que Pierre Bec nomme

${ }^{51}$ Ce document est cité par Michel Rousse dans son article « Mystères et farces à la fin du Moyen Âge, problèmes de théâtre populaire », La Scène et les tréteaux, Orléans, Paradigme, 2004, p. 248.

52 Jean-Claude AuBAILly, Le Monologue, le dialogue, et la sottie. Essai sur quelques genres dramatiques à la fin du Moyen Âge et au début du XVI siècle, Paris, 1976, p. 250. 
«distractions de clercs lettrés ${ }^{53}$. La constance de la dimension agonale rappelle que ces productions sont fondées sur l'esprit de compétition du jeu, mais la manière dont elles parodient les discours est tout à fait saisissante. Les jeux-partis sont jeux parce qu'ils jouent sur les règles de l'amour courtois, bien plus qu'ils n'y obéissent. Dans les trois débats amoureux de Christine de Pizan, ainsi que le relève l'éditrice Barbara Altmann ${ }^{54}$, l'auteur montre les désordres et les malheurs provoqués par « fol amour », subvertissant insensiblement l'idéal amoureux. Plus proche du jeu dramatique, le Dit des quatre offices de l'ostel du roi «à jouer par personnages » d'Eustache Deschamps reprend le principe de la confrontation qui est celui du débat, en le déplaçant dans le cadre familier des cuisines, pour que s'affrontent avec verdeur Panneterie, Eschançonnerie, Cuisine et Sausserie ${ }^{55}$ : entre jeu de société, jeu poétique et représentation théâtrale, la transition est ici insensible. Créer et récréer se trouvent indissociablement liés dans ces écrits nés dans et pour l'amusement d'une société qui goûte les raffinements savants des jeux poétiques. Replacés dans le contexte de la vie de cour, et pensés comme jeux, ces textes semblent peut-être se réduire à des œuvrettes de circonstance, mais ils s'inscrivent en fait dans cet élan ludique qui emporte la parole poétique vers la performance théâtrale.

Dans le jeu, la règle se situe au cœur du processus de création : le refrain de la ballade d'Eustache Deschamps le dit, même si le terme ne s'applique pas au jeu dans le poème: «on ne peut bien sans regle

53 Pierre BEC, La Joute poétique, De la tenson médiévale aux débats chantés traditionnels, Paris, Les Belles Lettres, 2000, p. 21.

${ }^{54}$ Barbara Altmann, The Love Debate poems of Christine de Pizan, op.cit. Cette édition comprend les trois débats de Christine de Pizan : Le Livre du Debat de deux amans, Le Livre des Trois jugemens, Le Livre du Dit de Poissy. Voir notamment l'introduction p. 28-31.

55 Eustache Deschamps, Dit des quatre offices de l'ostel du roi, in Cuvres complètes de Eustache Deschamps, éd. cit., tome VII, p. 175-192. 
ouvrer ${ }^{56}$. Cela suppose peut-être que le jeu impose sa règle à l'écriture, qui se plie au désir du prince et aux plaisirs de la cour. Bien souvent le jeu s'organise selon le principe du procès fictif, la cour seigneuriale devenant cour de justice. La cour amoureuse dite « de Charles VI», jeu de société auquel la cour parisienne se livre «par manière de passetemps », instaure par exemple un «jeu de rôle $»^{57}$ en assignant à des grands seigneurs des titres et des fonctions dans une cour censée réglementer les comportements amoureux. L'institution se dote d'une « charte » écrite, et pour les besoins du jeu prescrit la composition d'un certain type de poèmes :

« (...) [les] escriptures seront leues ainsy que ordonné sera et aprés seront baillés, toutes seelees, es mains de noz amoureux Presidens ou de l'un d'eulx, pour en déterminer et décider la sentence amoureuse ainsy que le caz requerra (...). $»^{58}$

La fiction de la cour de justice impose aux membres de l'institution courtoise une gestuelle, et suppose, pour l'auteur qui soumet son texte, de transposer dans l'écriture les règles du procès : exposition d'un cas, débats contradictoires et sentence rendue ou sollicitée auprès d'un juge forment la trame des jeux-partis, des débats et des jugements, des Arrêts d'Amour de Martial d'Auvergne. Ces écrits destinés au jeu ou susceptibles de s'y prêter comportent la possibilité d'une distribution des rôles, d'une gestuelle, d'un ton que les participants pourront identifier. La règle joue ici selon le principe de la parodie, comme imitation décalée de paroles et éventuellement de gestes codifiés.

Le principe du jeu laisse place à l'improvisation, à l'invention. Dans les jeux de société ou dans les jeux sportifs, les règles évoluent avec les joueurs, comme le montre l'exemple du jeu de Paume. Si le prince, par

\footnotetext{
${ }^{56}$ Ibid., p. 203.

57 Jacqueline CERQuiglini-Toulet, La Couleur de la mélancolie : la fréquentation des livres au XIV siècle, 1300-1415, Hatier, Paris, 1993, p. 49 sqq.

58 Carla Bozzolo, La Cour amoureuse dite de Charles VI, Paris, Le Léopard d'or, 1982, tome 1, p. 41, 1. 260-263.
} 
décision explicite, fixe le poids des balles et détermine les normes de leur confection, ce sont les joueurs qui ont introduit l'utilisation des raquettes et recommandé l'utilisation d'une corde sans barbules pour plus de précision. Ces dispositions sont rapidement adoptées dans les salles bourgeoises ${ }^{59}$, puis un traité de règles est élaboré au $\mathrm{XVI}^{\mathrm{e}}$ siècle dans les milieux des cours italiennes. Cette évolution montre bien que le processus de régulation est double et que les contraintes extérieures ne condamnent pas un jeu à l'immobilité, et se conçoivent plutôt comme un point de départ. L'invention de la règle signale cependant que l'on est passé de la recherche du plaisir à celle de la performance, du jeu au sport.

En ce sens le jeu est aussi le lieu privilégié d'une prise de conscience des règles: par la parodie, procès fictifs, débats pour rire, disputes décalées, plaintes amoureuses comiques ou faux échanges dévoilent les règles implicites qui structurent les comportements et les paroles. Les joueurs, selon l'expression employée par Michèle Gally pour les trouvères arrageois, «travaillent sur du déjà dit » ${ }^{60}$. Héritiers d'une pratique, d'une poétique, ils se l'approprient en la détournant. Les divertissements des clercs du parlement de Paris, dits clercs de la Basoche, qui débattent de « causes grasses » les jours de mardi gras, sont des jeux à la limite du sérieux : le clerc joue un procès pour rire, mais fait montre de son talent et de son habileté aussi bien que dans un procès sérieux, devant le public des maîtres. Le jeu, d'après l'étude menée par Marie Bouhaïk-Gironès ${ }^{61}$, est l'un des moyens par lesquels les jeunes apprentis intériorisent les règles de la justice et apprennent leur métier.

59 «Parce qu'il est noble et ostentatoire, ce jeu s'offre à l'imitation des catégories sociales désireuses de manifester leur place dans la société », Jean-Michel MEHL, Les Jeux au royaume de France, op. cit., p. 467.

${ }^{60}$ Michèle GALly, Parler d'amour au puy d'Arras, op. cit., p. 14.

${ }^{61}$ Marie BouhaïK-Girones, Les Clercs de la Basoche et le théâtre comique (Paris, 1420-1550), Paris, Honoré Champion, 2007. 
Entre jouer aux échecs, jouer un rôle et jouer un personnage, il existe une continuité de pratiques : au cœur d'un groupe, le joueur fait semblant, pour se divertir, d'être un autre. Des règles précises existent, et peuvent être reconstituées, mais il est frappant de voir que la réglementation du jeu est bien souvent imposée de l'extérieur, et que la conscience que des règles internes guident le cours du jeu ne se fait jour que progressivement, dans la pratique ludique elle-même. Période de fixation des règles du jeu, le Moyen Âge demeure aujourd'hui important dans l'imaginaire ludique : jeux de rôle, de société, en réseaux sont souvent très inspirés par cette période $^{62}$.

${ }^{62}$ Un colloque organisé à Bordeaux en avril 2008, et intitulé «Le Moyen Âge en jeu » témoigne de l'intérêt suscité par ces réactivations médiévalisantes du jeu. Voir la prépublication des actes de ce colloque en ligne: http://lapril.ubordeaux3.fr/spip.php?breve67 


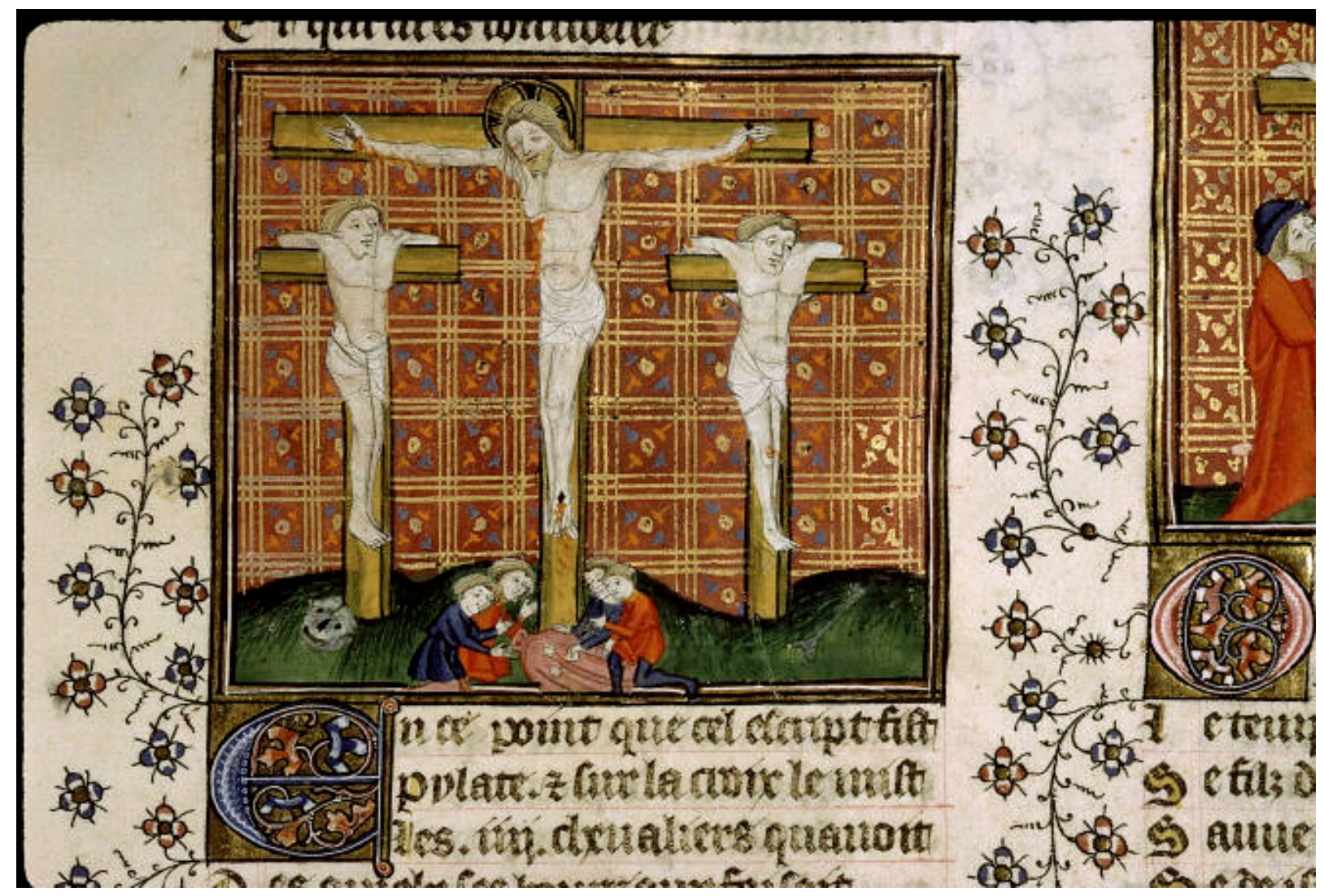

Illustration 1 : Le tirage au sort des vêtements du Christ,

Paris, Bibliothèque Sainte-Geneviève, ms. 1130

(Guillaume de Digulleville, Le Pelerinage de Jesus Christ), f 214. 


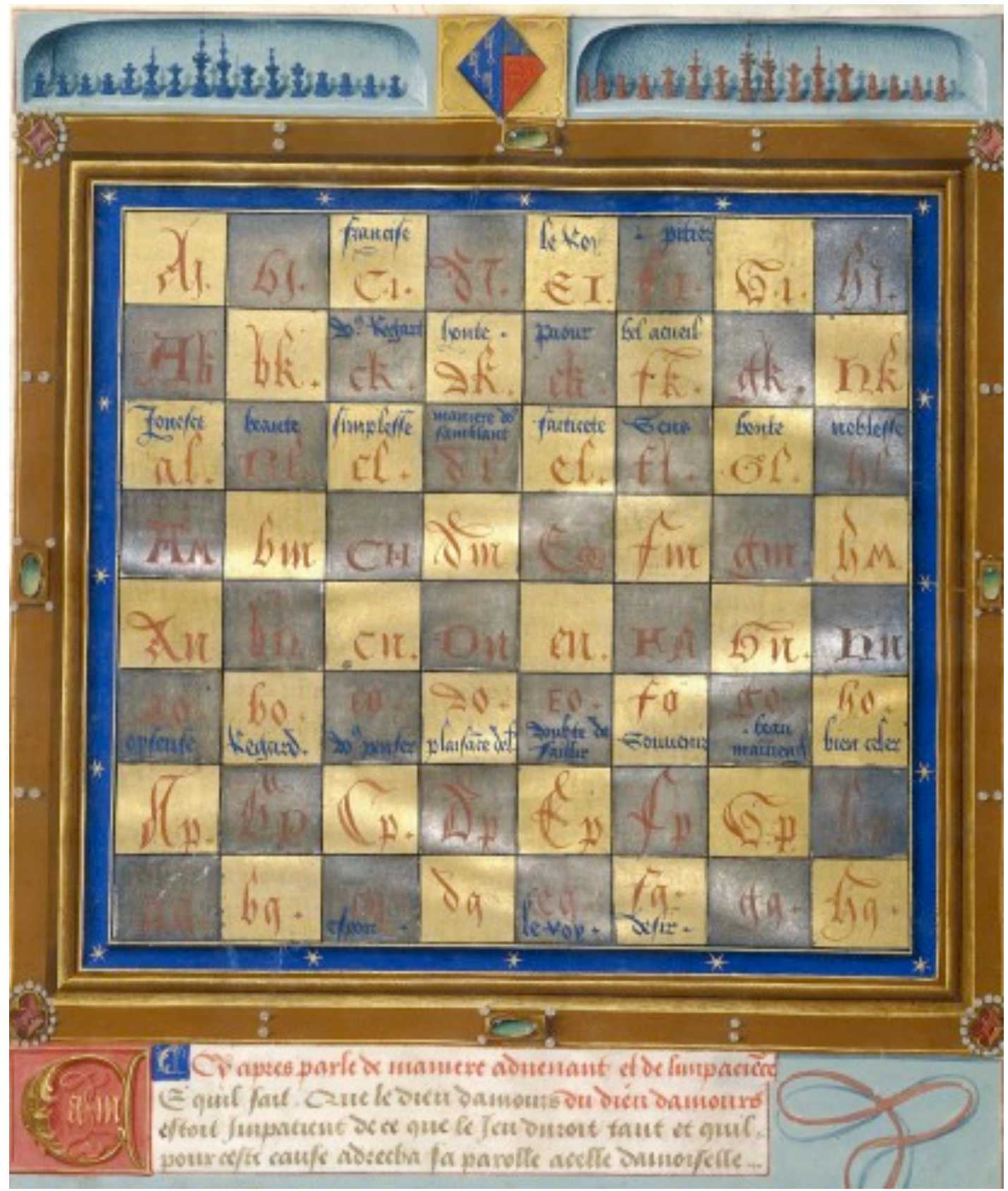

Illustration 2 : L'échiquier d'amour du Livre des eschez amoureux moralisés d'Évrart de Conty, BnF, fr. 9197, f 437. 